\title{
Diabetes Mellitus Specific Infections
}

(BIRDEM Med J 2017; 7(1):1-5)

People with diabetes mellitus are exceptionally prone to develop infections like Emphysematous pyelonephritis (EPN), Mucormycosis, Malignant otitis externa and Melioidosis. Birdem general hospital is 700 bed tertiary care multidisciplinary hospital in Dhaka, Bangladesh with around $80 \%$ patients having Diabetes Mellitus. We have series of case with Melioidosis, Emphysematous pyelonephritis, Mucormycosis and Malignant otitis externa. Majority of these cases were detected and treated in our hospiatal during last few years. These cases were reported to journal, presented to national and internationational seminer. We ,not infrequently encounter these type of cases because of professional involvement in this hospital dedicated to people with Diabetes mellitus.

\section{Mucormycosis}

(previously called zygomycosis) - is a fungal infection, can affect any organ but commonly affect the sinuses, brain, or lungs mostly in people with a weakened immune system, caused by a mold of mucormycete group frequently found in the soil and in dying plants.

People affected: Diabetes (usually poorly controlled), AIDS, burns , metabolic acidosis(diabetic ketoacidosis), leukemia and lymphoma etc.

Why Diabetes Mellitus (DM) preponderance:The fungus develop in environment of high glucose. The fungus grows in acid environment.Most of the series shows Mucormycosis commoner in patients with Diabetis ketoacidosis at presentation.

Site of involvement: Rhino Orbito Cerebral: Sinus ,orbit and brain infection, which may start as a sinus infection, ultimately causes swelling of the cranial nerves.

Pulmonary Mucormicosis: Pneumonia that gets worse quickly and may spread to the chest cavity, heart and brain.

Mucormicosis of the gastrointestinal tract, skin, and kidney may happen.
Symptoms /signs: According to site of involvement. Commonest site Rhino-orbito-cerebral.

Symptoms of rhinoorbito cerebral Mucormicosis include: Eye swelling and protrusion (proptosis), Dark nasal Escher (scabbing), Fever, Headache, Mental status changes, Redness of skin overlying sinuses, Sinus pain or congestion. These are in addition to original disease viz Diabeteic ketoacidosis

Clinical Examination :An ear-nose-throat (ENT) doctor and an eye specialist should see the patient in case of rhioorbital and neurologist if cerebral manifestation is there.

Investigations:Testing depends on symptoms but may include: CT or MRI scans. A biopsy must be done to make a definitive diagnosis of Mucomycosis

\section{Treatment}

Surgery should be done immediately to remove all dead and infected tissue. Surgery can lead to disfiguration because it may involve removal of the palate, parts of the nose, or parts of the eye. Without such aggressive surgery, however, chances of survival are greatly decreased.

Systemic antifungal medicines: Usually amphotericin $\mathrm{B}$ is needed.

\section{Prognosis}

Mucormycosis has an extremely high death rate even when aggressive surgery is done. Death rates range from $25-85 \%$ depending on the body area involved and overall health.

\section{Malignant otitis externa}

Malignant external otitis (MEO) is an infection that affects the external auditory canal and temporal bone. The causative organism is usually Pseudomonas aeruginosa, and the disease commonly manifests in 
elderly patients with diabetes. The infection begins as an external otitis that progress into an osteomyelitis of the temporal bone.

Comorbid conditions: Patients with $\mathrm{MEO}$ almost always have diabetes( $>90 \%)$, MEO has been reported in all age groups but is most common in patients who are elderly (age $>60 \mathrm{y}$ ).

\section{Clinical features}

Symptom: Severe, unrelenting, deep-seated otalgia,Temporal headaches, Purulent otorrhea. Possible dysphagia, hoarseness, and/or facial nerve dysfunction. The pain is out of proportion to the physical examination findings.

Sign: Inflammatory changes are observed in the external auditory canal and the periauricular soft tissue. Marked tenderness is present in the soft tissue between the mandible ramus and mastoid tip. Granulation tissue is present at the floor of the Osseo cartilaginous junction. This finding is virtually pathognomonic of malignant external otitis. Otoscopic examination may also reveal exposed bone.

\section{Cranial neuropathy}

Cranial nerves can be affected by inflammation along the skull base or by a neurotoxin produced by Pseudomonas species. The facial nerve (VII) is affected most commonly, usually at the stylomastoid foramen. IX, X, and XI can be affected also XII.The cranial nerves (V-XII) should be examined

Intracranial complications: These complications rarely occur in the absence of cranial nerve palsies. Meningitis, brain abscess, and dural sinus thrombosis may ensue. Mental status examination should be performed. Deterioration of the mental status may indicate intracranial complication. The tympanic membrane is usually intact. Fever is uncommon.

\section{Causes}

Diabetes is the most significant risk factor for developing malignant external otitis.

- Small-vessel vasculopathy and immune dysfunction associated with diabetes are primarily responsible for this predisposition. The cerumen of patients with diabetes has a higher $\mathrm{pH}$ and reduced concentration of lysozyme, which may impair local antibacterial activity. No difference in predisposition is found between diabetes types I and II. The predisposition is not necessarily related to the severity of glucose intolerance or periods of hyperglycemia. The term malignant is used to emphasize the serious nature of this infection. Some series claimed their $100 \%$ cases to be with DM.

Aural irrigation: As many as $50 \%$ of cases of malignant external otitis have been reported to be preceded by traumatic aural irrigation in patients with diabetes.

Patients with Immunodeficiencies, such as lymphoproliferative disorders or medication-related immunosuppression, AIDS may have predilection of it.

\section{Laboratory evaluation}

The leukocyte count in MEO is usually normal or mildly elevated.The erythrocyte sedimentation rate (ESR) is invariably elevated,. ESR can be used to support the clinical diagnosis since acute external otitis or ear canal malignancy usually does not cause much elevation of ESR

Biochemistry: Patients without a history of diabetes should be tested for glucose intolerance.

Culture and sensitivities from the external auditory canal drainage should be performed ideally before antimicrobial therapy is initiated. The most common causative organism is $P$. aeruginosa (95\%). Less common organisms identified include Aspergillus and Proteus species, Candida species, Staphylococcus aureus (Specially MRSA), and S epidermidis.

CT scanning and MRI are both useful for evaluating the anatomic extent of soft tissue inflammation, abscess formation, and intracranial complications.

A biopsy of the external auditory canal to exclude carcinoma or other etiologiesis needed.

\section{Treatment and management}

Treatment includes meticulous glucose control, aural toilet, systemic and ototopic antimicrobial. 


\section{Duration of therapy}

Symptoms and examination findings improve with appropriate therapy, but these changes do not correlate with the length of needed therapy. Despite symptom relief, prolonged antimicrobial treatment as indicated for osteomyelitis is still indicated Treatment response should be evaluated with a gallium citrate Ga 67 scan every 4-6 weeks during treatment.

Surgery is now reserved for local debridement, removal of bony sequestrum, or abscess drainage.

\section{Outlook (Prognosis)}

Majority cases with Diabtes mellitus have frustrating outcome.Malignant otitis externat often responds to long-term treatment, especially if treated early. It may return in the future. Severe cases may be deadly.

\section{Emphysematous pyelonephritis}

Emphysematous pyelonephritis (EPN) is a severe, necrotizing infection of the renal parenchyma; it causes gas formation within the collecting system, renal parenchyma, and/or perirenal tissues, Other terms that have been used to describe the condition include renal emphysema and pneumonephritis. Gas in the renal pelvis alone, without parenchymal gas, is often referred to as emphysematous pyelitis. EPN is common in persons with diabetes(some series claims their $100 \%$ cases were with DM) often has a fulminating course, and can be fatal if not recognized and treated promptly

Definition: Presence of culture positive UTI and presence of gas in the renal parenchyma / collecting system as evident on USG of KUB then confirmed by CT scan

\section{Signs and symptoms}

Typical presenting features of EPN include the following: Fever (79\%, Abdominal or flank pain (71\%), Nausea and vomiting (17\%), Dyspnea (13\%), Altered sensorium (19\%), Shock (29\%).

\section{Other possible findings include the following}

Crepitus over the flank area in advanced cases of EPN, Pneumaturia is uncommon unless emphysematous cystitis is present, Subcutaneous emphysema and pneumomediastinum have been reported in a case of EPN,
Comorbidities include alcoholism, malnourishment, renal calculi, and diabetic ketoacidosis.

\section{Infectious agents}

Among the bacteria associated with emphysematous pyelonephritis, Escherichia coli is isolated in $66 \%$ of patients, and Klebsiella species are reported in $26 \%$ of patients. Proteus, Pseudomonas, and Streptococcus species are other organisms found in patients with EPN, and Entamoeba histolytica and Aspergillus fumigatus also have been reported to cause the disease. Mixed organisms are observed in $10 \%$ of patients. Positive blood culture results are identical to urine culture results in $54 \%$ of patients. Rare organisms such as Clostridium and Candida species have also been isolated in patients with EPN.

\section{Diagnostic workup}

A high index of suspicion is important when attempting to diagnose emphysematous pyelonephritis promptly.

Laboratory data reveal leukocytosis with a left shift, pyuria, infected urine, thrombocytopenia, an elevated creatinine level, and positive blood culture. Patients with urosepsis and shock should undergo cardiac and pulmonary function assessment as needed.

Ultrasonography:Often reveal high echogenic areas with dirty shadowing. Hydronephrosis and perinephric fluid may also be seen:

Computed tomography (CT) scanning is the definitive imaging test for EPN

Several patterns have been described, including streaky, streaky and mottled, and streaky and bubbly. Gas can be rim like or crescent shaped in the perinephric area. Gas can also be seen in the renal vein or inferior vena cava and along the psoas muscle. Perinephric abscess may lead to significant gas accumulation in the perinephric space. A stone may be seen in the collecting system.

Staging and its evolution according to imaging characteristics: 


\section{Management:}

Management algorhythm for EPN

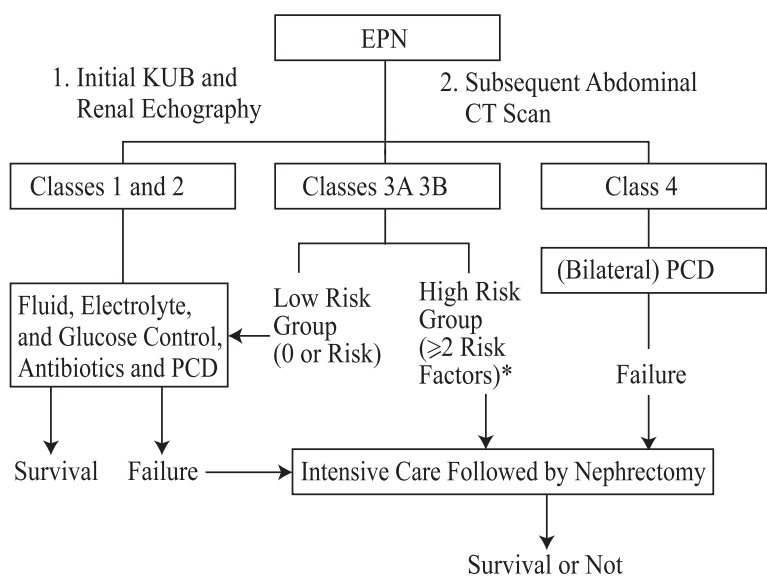

Patients with emphysematous pyelonephritis should be treated with aggressive medical management and, possibly, prompt surgical intervention. However, surgical intervention should be performed only after stabilization of the patient's cardiorespiratory status. Prompt hydration, fluid resuscitation, and systemic antibiotics are the mainstays of medical management in EPN. Initial antibiotic therapy is based on local resistance patterns and patient factors; agents may include intravenous ampicillin, third- or fourthgeneration cephalosporins, gentamicin, amikacin, vancomycin, and metronidazole. Regimens are adjusted as necessary when culture sensitivities become available. In patients with renal compromise, doses must be adjusted according to creatinine clearance.A monitoredcare facility may be needed for patients in shock. Control of diabetes and maintenance of adequate fluid balance should be achieved quickly. Any obstruction found on imaging studies should be relieved with either percutaneous drainage or stent placement. The presence of a stone often leads to obstruction, which must be urgently relieved with percutaneous drainage or stenting. Definitive treatment for stones should be deferred until later.

\section{Conservative treatment using percutaneous drainage with antibiotics is indicated as follows}

1. Patients with compromised renal function 2. Early cases associated with gas in the collecting system alone and patient is in otherwise in stable condition 3. Class 1 and class 2 EPN 4. Class 3 and class 4
EPN - In the presence of fewer than 2 risk factors (eg, thrombocytopenia, elevated serum creatinine levels, altered sensorium, shock)

The use of nephrectomy is indicated as follows

1. No access to percutaneous drainage or internal stenting (after patient is stabilized) 2.Gas in the renal parenchyma or "dry-type" EPN 3.Possibly bilateral nephrectomy in patients with bilateral EPN 4.Class 3 and class 4 EPN - In the presence of more than 2 risk factors (eg, thrombocytopenia, elevated serum creatinine, altered sensorium, shock)

\section{Prognosis}

Overall EPN mortality rate of $19-40 \%$. Reported treatment success rates with percutaneous drainage and antibiotics (66\%) and with nephrectomy (90\%). Furthermore, the investigators reported a high risk of death in patients with serum creatinine levels greater than $1.4 \mathrm{mg} / \mathrm{dL}$ and platelet count of less than $60,000 / \mu \mathrm{L}$.

\section{Gas and it's mechanism of production}

Schainuck et al proposed that fermentation products from tissue necrosis produced carbon dioxide. Three investigators analyzed the gas content, and all 3 demonstrated that the major components of the gas in EPN include nitrogen (60\%), hydrogen $(15 \%)$, carbon dioxide $(5 \%)$, and oxygen $(8 \%)$.

Huang et al concluded that mixed acid fermentation is the mechanism of gas production based on the presence of hydrogen.

In presence of high glucose-in DM, the glucose fermenting bacteria produces gas.

Impaired host immunity, a perfect situation for bacteria to grow.It has been suggested that gas-forming infections depend on rapid tissue catabolism and impaired transport of the end products at the inflammatory site. Diabetic microangiopathy may also contribute to the slow transport of catabolic products and may lead to accumulation of gas.

\section{Melioidosis}

Whitmore's disease is an infectious disease caused by a Gram-negative bacterium, Burkholderia pseudomallei, found in soil and water. It is of public health importance in endemic areas (south and southeast Asia and northern Australia).It exists in acute and chronic forms.. It has 
the potential for epidemic spread to areas where it is not endemic, and sporadic case reports elsewhere in the world suggest that as-yet-unrecognized foci of infection may exist. Environmental determinants of this infection, apart from a close association with rainfall, are yet to be elucidated. The sequencing of the genome of a strain of B. pseudomallei has recently been completed and will help in the further identification of virulence factors. The presence of specific risk factors for infection, such as diabetes mellitus suggests that functional neutrophil defects are important in the pathogenesis of melioidosis; others have defined virulence factors (including a type III secretion system) that allow evasion of killing mechanisms by phagocytes. There is a possible role for cell-mediated immunity, but repeated environmental exposure does not elicit protective humoral or cellular immunity. A vaccine is under development, but economic constraints may make vaccination an unrealistic option for many regions of endemicity.
Disease manifestations are protean; diagnosis relies on culture of the organism. Despite the introduction of ceftazidime- and carbapenem-based intravenous treatments, melioidosis is still associated with a significant mortality attributable to severe sepsis and its complications. A long course of oral eradication therapy is required to prevent relapse. Studies exploring the role of preventative measures, earlier clinical identification, and better management of severe sepsis are required to reduce the burden of this disease.Most of the series claimed almost $80 \%$ of their cases were with DM.The exact mechanism or pathogenesis of relation to diabetes Mellitus is yet to be revealed.

The topic was discussed in our journal as editorial (Melioidosis: an emerging infection in Bangladesh .Birdem med j 2016;6:3-6).

\section{Khwaja Nazim Uddin}

Professor of Medicine

BIRDEM General Hospital, Dhaka 